Rev. salud pública. 14 (2): 248-259, 2012

\title{
Impacto de indicadores de gestión en salud sobre estrategias de un servicio de salud público
}

\section{The impact of health management indicators on a public health service's strategies}

\author{
Patricia C. Huerta-Riveros ${ }^{1}$, Iván R. Paúl-Espinoza ${ }^{2}$ y Carolina E. Leyton-Pavez ${ }^{3}$ \\ 1 Universidad del Bío Bío. Concepción, Chile. phuerta@ubiobio.cl \\ 2 Hospital Clínico Herminda Martin. Chillán, Chile. ivanrenato2001@yahoo.com \\ 3 Servicio de Salud Nuble. Chillán-Chile. carolina.leyton@redsalud.gov.cl
}

Recibido 7 Febrero 2012/Enviado para Modificación: 14 Marzo 2012/Aceptado 12 Abril 2012

\section{RESUMEN}

Objetivos Realizar un análisis del impacto de los indicadores de gestión en salud sobre las estrategias que implementa un Servicio de Salud Pública.

Métodos Se realiza un estudio a un Servicio de Salud Pública en Chile analizando los indicadores de gestión relevantes, a través de un análisis longitudinal, para el periodo 2006-2010.

Resultados Los resultados del estudio demuestran que los indicadores de gestión en salud direccionan las estrategias utilizadas por los directivos que pertenecen a una Red Asistencial de Salud Pública.

Conclusión Las estrategias adoptadas por los directivos deben estar guiadas por el análisis oportuno a los indicadores de gestión en salud, con la finalidad de brindar una atención de calidad a los usuarios de una Red Asistencial de Salud Pública.

Palabras Clave: Indicadores de servicios, estrategias, salud pública, servicios de salud (fuente: DeCS, BIREME).

\section{ABSTRACT}

Objectives Analysing the impact of health management indicators on a public health service's strategies.

Methods Studying a Chilean public healthcare service's strategies (2006-2010) by analysing key management indicators using longitudinal data analysis.

Results The results of the study demonstrated that health management indicators guided the strategies being used by a public Chilean healthcare network's managers. Conclusions The strategies adopted by the managers should be guided by early analysis of healthcare management indicators for providing quality healthcare attention for the users of a public healthcare network. 
Key Words: Health service indicators, strategy, public health, healthcare service (fuente: DeCS, BIREME).

L a salud y el acceso a los servicios relacionados constituyen un derecho humano y, como tal, una preocupación de la sociedad que debe ser asumida desde una perspectiva intersectorial (1). En este sentido, los problemas de salud involucran soluciones más amplias que el control y/o curación de enfermedades que deben ser financiadas.

Por ello, el Ministerio de Salud (MINSAL) anualmente determina los presupuestos del Sector Salud. Estos presupuestos cubren fundamentalmente, los gastos de los establecimientos de salud pertenecientes a los Servicios de Salud Pública y se distribuyen principalmente en tres partidas presupuestarias: gastos en personal, bienes y servicios de consumo e inversión. Donde más de dos tercios de los fondos transferidos a cada Servicio de Salud cubren los gastos generados por los hospitales, independiente de la actividad que generan $(2,3)$.

Por esta razón, en losúltimos años elFondo Nacional de Salud(FONASA) ha realizado un esfuerzo sistemático para clarificar las transferencias a los Servicios de Salud y para ello ha definido, respecto de los mismos, brechas de equidad y eficiencia, que son utilizadas actualmente, para corregir eventuales distorsiones históricas producidas en la distribución de los fondos, debido a cambios en las condiciones socioeconómicas de la población, fenómenos migratorios, mejorías ambientales, nuevos establecimientos de salud, entre otros $(4,5)$.

Considerando el contexto anterior, se vuelve fundamental analizar los indicadores de gestión que permitan un uso más eficiente de los recursos asignados a los Servicios de Salud y que, por ende, direccionan las estrategias en el ámbito financiero, asistencial y de recurso humano que se implementan posteriormente.

Con base en ello, el presente artículo posee como objetivo analizar el impacto de los indicadores de gestión en salud sobre las estrategias que implementa un Servicio de Salud Pública. Para ello, se realizó un estudio al Servicio de Salud de Nuble en Chile analizando los indicadores de gestión relevantes, a través de un análisis longitudinal, para el periodo 2006-2010. 


\section{MÉTODOS}

Los siguientes antecedentes entregan el contexto sobre el cual se levantan los indicadores y las estrategias del Servicio de Salud Nuble. En particular, la Provincia de Ñuble posee 21 comunas y otorga atención en salud a 380312 personas, a través de 106 establecimientos que forman parte del Servicio de Salud Ñuble.

En concreto, las comunas de la Provincia de Ñuble que concentran el mayor número de población corresponden a Chillán con 38,3\%, seguida de San Carlos con 11,1 \% y la tercera en importancia es Chillán Viejo con un $6,5 \%$, sumando el $56 \%$ del total de la provincia.

A la vez, el índice de envejecimiento de la provincia de Ñuble es de un $41,4 \%$, es decir, que por cada 100 menores de 15 años existen 41 adultos mayores. La Ruralidad de la provincia alcanza el 34,9 \% y la Población desocupada es de $8,3 \%$. El porcentaje de analfabetismo es de $12,1 \%$, muy superior al país que es de un 3,9\%.

Los años de escolaridad en Ñuble alcanzan a los 8 años y en el país a 10,1 años. Además, la población de Ñuble registra un porcentaje de discapacidad de 9,0\%, superior a la región del Bío Bío, que es de un 7,5 $\%$. La tasa de mortalidad en menores de 5 años en la provincia de Ñuble es significativamente más elevada que la tasa de la región y el país. Sin embargo, en la Provincia de Ñuble se viene observando una tendencia a la disminución de los años de vida potencialmente perdidos (AVPP), en el quinquenio 1994 al 1998 el AVPP fue de 102,2 en el quinquenio 1999-2003 fue de 98,2 y el quinquenio 2002- 2006 fue de 93,6 años. Por último, la Provincia de Ñuble presenta tasas significativamente más elevadas en mortalidad por enfermedad isquémica del corazón, cerebrovasculares y diabetes, que la región y el país, siendo la primera causa de muerte el accidente cerebrovascular (75,9 x 100 000). Sumado a lo anterior, la tasa de mortalidad por accidentes de tránsito en la provincia de Ñuble se encuentra por sobre la región y el país (6).

Lo anterior, mediante un análisis de la situación financiera, asistencial y de recursos humanos consolidada, de cada uno de los hospitales y demás establecimientos de su dependencia, información que trimestralmente es remitida al Ministerio de Salud $(7,8)$. De esta forma, se busca observar las estrategias adoptadas por los directivos, que permiten brindar una 
atención de calidad a los usuarios de una Red Asistencial de Salud Pública, dependiendo de los indicadores reportados.

\section{Indicadores de Gestión en Salud}

En términos generales, los indicadores de salud representan medidas resumidas que capturan información relevante sobre distintos atributos y dimensiones del estado de salud y del desempeño del sistema de salud. Específicamente, proporcionan señales para monitorear la gestión de un servicio, lo que permite asegurar que las actividades vayan en el sentido correcto, evaluar los resultados de una gestión frente a sus objetivos, metas y responsabilidades (9). Por lo tanto, es la expresión cuantitativa del comportamiento y desempeño de un proceso, cuya magnitud, al ser comparada con algún nivel de referencia, puede estar señalando una desviación sobre la cual se toman acciones correctivas o preventivas (10).

Debido a su relevancia e impacto en la población, todo indicador de gestión en salud debiera ser considerado por los directivos de las redes asistenciales para guiar las estrategias a implementar en los establecimientos que conforman la Red Asistencial de Salud Pública.

En particular, no es necesario tener bajo control continuo muchos indicadores, sino sólo los más importantes, es decir, los claves (11). Entre los diversos indicadores de gestión que puede proporcionar un Servicio de Salud (12), se tiene:

a. Indicadores Asistenciales: Con la finalidad de presentar el producto o resultado de la actividad asistencial de los Servicios de Salud, se ha escogido, de los registros estadísticos elaborados por los Servicios, una serie de indicadores que dan cuenta de la mayor parte de las actividades de los establecimientos de la red hospitalaria. Estos son:

- Índice Ocupacional: Este indicador, informa del porcentaje de utilización del recurso hospitalario "cama". Representa la proporción de camas que se ocuparon con relación a las camas disponibles en un periodo. Este indicador se presenta acumulado durante el año.

- Promedio de Días de Estancia: Este indicador, reporta el número de días cama promedio acumulados ocupados por cada egreso hospitalario durante el periodo observado. Para efectos del análisis, sólo se consideran los establecimientos hospitalarios con datos acumulados durante los trimestres. 
- Número de Consultas de Especialidad: Este indicador, expresa el número de Consultas de Especialidad efectivamente realizadas en un periodo acumulado del año. Para efectos del análisis se consideran los establecimientos hospitalarios y Centros de Resolución de Especialidades, Centro de Diagnóstico y Tratamiento (CDT) y Centro de Referencia en Salud (CRS).

- Porcentaje de Consultas Nuevas de Especialidad: El porcentaje de Consultas Nuevas de Especialidad corresponde al número de Consultas Nuevas de Especialidad del Total de Consultas de Especialidad en un periodo de tiempo. Este indicador debe medirse acumulado y para efectos del análisis se consideran los establecimientos hospitalarios.

- Porcentaje de Cesáreas sobre Partos: Muestra la relación existente entre los partos vaginalesy cesáreas, del total de partos atendidos en losestablecimientos de la Red Asistencial. Este indicador debe medirse acumulado.

- Número de Intervenciones Quirúrgicas electivas: Este indicador, reporta el número total de Intervenciones Quirúrgicas mayores y menores efectivamente realizadas en un periodo acumulado del año. Este indicador debe medirse acumulado.

b. Indicadores de Gestión Financiera: Los indicadores de Gestión Financiera informan del comportamiento de la información relativa a ingresos y gastos percibidos y devengados, grado de ejecución presupuestaria y situación de la deuda. Para realizar un análisis crítico que permita extraer conclusiones que reflejen efectivamente la gestión financiera de la Red Asistencial, es necesario observar el proceso anual completo, es decir, los datos acumulados del año. Completado el ejercicio contablepresupuestario anual, se podrá verificar la eficiencia de la recuperación de ingresos, la capacidad de enfrentar el presupuesto anual y el rendimiento del gasto. Por lo anterior, en este apartado se presentarán las definiciones y las fórmulas de cálculo que se están utilizando.

- Ingresos de Operación Devengados: Este indicador expresa la totalidad de los ingresos de operación devengados de los Servicios de Salud.

- Porcentaje de Ingresos de operación sobre total Devengados: Indica la proporción de los ingresos de operación Subtítulo 07 por venta de Servicios (ingresos propios) sobre la totalidad de los ingresos de operación devengados.

- Porcentaje de Ejecución del Subtítulo 21: Este indicador muestra qué proporción del presupuesto asignado al subtítulo 21 ha sido ejecutado durante el periodo analizado acumulado. 
- Porcentaje de Deuda sobre Subtítulo 22: Este indicador muestra la proporción del gasto total generado y devengado en el periodo acumulado evaluado en bienes y servicios de consumo que no están pagado.

- Porcentaje de Ejecución del Subtítulo 22: Este indicador muestra qué proporción del presupuesto asignado al subtítulo 22 ha sido ejecutado durante el periodo analizado acumulado.

- Indicador de Rendimiento del Gasto Operacional: Este indicador muestra la relación entre la producción valorizada (la facturación se obtiene a partir de la valorización de las actividades que se realizan en los Servicios de Salud, sin considerar la compra de servicios a terceros) y el gasto operacional, es decir, la relación entre lo que produce cada establecimiento y el Servicio de Salud en su conjunto, por cada peso gastado en la operación del mismo.

- Diferencia Ingresos y Gastos Devengados de Operación: Indica un mayor ingreso o gasto en el periodo evaluado.

- Concentración de la Deuda Hospitalaria del Subtítulo 22, 29, 34: La concentración de la Deuda Hospitalaria hace referencia a la sumatoria de los Bienes y servicios de Consumo (Subtítulo 22), Adquisición de activos no financieros (Subtítulo 29) y servicios de la deuda (Subtítulo 34) en el periodo acumulado, que no está pagado.

c. Indicadores de Recursos Humanos: La información utilizada para el análisis es la entregada directamente por los Servicios de Salud y recoge los datos desagregados por establecimiento bajo dependencia directa, así como de la dirección respectiva, como estructura administrativa.

- Rendimiento de Recursos Humanos en Personas: Este indicador muestra cuanto de los ingresos vía producción en promedio genera cada persona en el periodo evaluado.

- Rendimiento de Recursos Humanos en Horas Semanales: Este indicador muestra cuanto de los ingresos vía producción en promedio genera la dotación efectiva en horas semanales en el periodo evaluado.

- Número de Días de Licencia Sobre Dotación Efectiva: El índice de ausentismo corresponde al número de días de licencia médica sobre dotación d personal (número de personas) no incluye licencias médicas curativas maternales, ni licencias por enfermedades de trabajo.

Población y muestra

La población objeto de estudio son los establecimientos de Salud Pública en Chile. La muestra utilizada se encuentra constituida por los 
establecimientos del Servicio de Salud Pública de Ñuble, que conformaban una red asistencial durante el periodo 2006-2010. Específicamente, la muestra está conformada por hospitales, Centros de Alta Especialidad (CAE), Centro de Salud Familiar (CESFAM), Centro Comunitario de Salud Familiar (CECOF), Servicio de Atención primaria de Urgencia (SAPU) y Postas. Las fuentes de información utilizadas fueron bases de datos oficiales que registran información del servicio de salud, tales como: bases de datos del Instituto Nacional de Estadísticas (INE), Ministerio de Salud (MINSAL) específicamente en el Departamento de Estadísticas e Información en Salud (DEIS), Ministerio de Hacienda, Ministerio de Desarrollo Social y Fondo Nacional de Salud (FONASA).

Ámbito de aplicación

El estudio se realizará en Chile por ser el país en el cual ha cobrado relevancia el seguimiento a los resultados alcanzados por los Servicios de Salud (13) y se analizará específicamente el Servicio de Salud Ñuble, el cual ha establecido una unidad de control de gestión encargada del seguimiento y evaluación de los indicadores de gestión en salud, entre otras tareas. Específicamente, el Servicio de Salud Ñuble es un organismo público que depende del Ministerio de Salud y de acuerdo al Reglamento Orgánico vigente "le corresponde la articulación, gestión y desarrollo de la Red Asistencial correspondiente, para la ejecución de las acciones integradas de fomento, protección y recuperación de la salud, como también la rehabilitación y cuidados paliativos de las personas enfermas".

\section{RESULTADOS}

A continuación se presentan los resultados de los indicadores de Gestión Asistencial, Indicadores de Gestión Financiera e Indicadores de Gestión de Recurso Humano, como se indica en la Tabla 1, Tabla 2 y Tabla 3.

Estrategias en salud

El monitoreo a los resultados de los indicadores presentados en la sección anterior generó que los establecimientos de la Red Asistencial del Servicio de Salud Nuble implementará las siguientes estrategias en beneficio de la población. 
Tabla 1. Indicadores de Gestión Asistencial

\begin{tabular}{|c|c|c|c|c|c|c|c|}
\hline & Indicador & 2006 & 2007 & 2008 & 2009 & 2010 & Promedio \\
\hline 1 & İndice Ocupacional (\%) & 70,7 & 70,8 & 70,8 & 70,4 & 72,4 & 71,0 \\
\hline 2 & Promedio de Días de Estada & 5,9 & 5,9 & 5,7 & 5,9 & 6,2 & 5,9 \\
\hline 3 & Intervalo de Sustitución & 2,5 & 2,4 & 2,4 & 2,5 & 2,4 & 2,4 \\
\hline 4 & $\begin{array}{l}\text { Porcentaje de cumplimiento de } \\
\text { consultas de especialidad (\%) }\end{array}$ & 86,6 & 55,3 & 112,5 & 118,0 & 143,6 & 103,2 \\
\hline 5 & Porcentaje de partos (\%) & 75,5 & 66,1 & 63,6 & 72,7 & 66,6 & 68,9 \\
\hline 6 & Porcentaje de cesáreas (\%) & 24,9 & 33,9 & 36,4 & 27,3 & 33,4 & 31,2 \\
\hline 7 & $\begin{array}{l}\text { Intervenciones quirúrgicas por } \\
\text { pabellón }\end{array}$ & 184,2 & 249,1 & 231,3 & 258,1 & 232,1 & 231 \\
\hline
\end{tabular}

Estrategias de Gestión Asistencial

- Call center y gestión no se presenta (NSP): oficina creada para evitar la pérdida de horas de especialidad que alcanzaba cerca de un $20 \%$, a través de llamadas telefónicas y un ágil mecanismo de reemplazo.

- Gestión de las horas médicas en relación a feriados, días administrativos, capacitaciones yotrasinasistencias médicas, para evitar el incumplimiento.

- Gestión de "altas médicas": gestión de la subdirección médica para agilizar y emitir normativas respecto a la oportunidad de las altas. Una vez producidas esta gestión administrativa y clínica para agilizar su concreción, permitiendo la reducción de días de estada y la oportuna disponibilidad de la cama (gran cantidad de hospitalizaciones por urgencias). Para ello se creó la Unidad de Cuidados al Alta (UCA), lugar físico para permanencia de los usuarios y coordinación con hospitales de menor complejidad y familiares.

- Gestión de optimización de pabellones quirúrgicos, con uso en la tarde y coordinación de los horarios de los diferentes actores.

- Implementación de programas especiales (locales) para compras de servicios quirúrgicos pero usando los pabellones, insumos y gastos fijos institucionales (a diferencia de la compra "total"). Orientados en dos vertientes: Acceso Universal a Garantías Explícita (AUGE) y brechas.

- Gestión médica con jefes de servicio pertinentes de "análisis y acción" respecto al porcentaje de cesáreas.

- Fuerte impulso desde la Provincia de Ñuble a la "Coordinación Regional de los Servicios de Salud", instancia prevista en el reglamento de los Servicios pero con bajo desarrollo hasta entonces, su objetivo fue hacer expedito el tránsito de usuarios en la red, de acuerdo a los diferentes niveles de complejidad, lo que incide fuertemente en objetivos sanitarios, satisfacción usuaria y también en promedio días estada, índice ocupacional. De hecho, Nuble y su Director coordinaron tal instancia desde su instauración en 2005 hasta agosto del año 2010. Además, se 
crearon comités clínicos regionales de las distintas especialidades (cardiología, oncología etc.) y comités de coordinación administrativa y de gestión (Servicio de Orientación Médica Estadística, AUGE, Informática etc.).

Estrategias de Gestión Financiera

- Compras centralizadas para los establecimientos del servicio, como una pequeña central de abastecimiento provincial, lo que permitió ahorro (deuda 22), abastecimiento más oportuno y mejor control.

- Separación de los departamentos de Recursos Físicos y Recursos Financieros, lo que permitió mejor control cruzado, ahorro y transparencia.

- Reacción de "comités de producción" en la Dirección de Servicio y en los establecimientos. Comité que controla la producción de prestaciones valoradas (PPV) e institucionales (PPI) y su relación con los presupuestos respectivos y su adecuada y oportuna ejecución. Se evitan subproducciones de lo comprometido lo que conlleva devolución de presupuesto ya recibido duodecimalmente, con las consecuencias que ello tiene sobre los indicadores y se tiende a evitar también sobreproducciones no financiadas que afectan los indicadores.

Estrategia de Gestión de Recursos Humanos

- Funcionamiento de "Comités de licencias" en los establecimientos a fin no sólo de fiscalizar los subsidios de incapacidad laboral, sino de analizar las razones que inciden en ellos para evitarlos.

- Creación de una función específica de acompañamiento al personal, en el subdepartamento de gestión de las personas, que generó una política de salud del personal, con exámenes preventivos, policlínicos del personal y algunos programas especiales de salud (por ejemplo salud odontológica en convenio con bienestar).

- Planes anuales de capacitación del personal, con énfasis en la gestión por competencias y comienzo del desarrollo de un sistema de evaluación de las capacitaciones en relación a su impacto medible sobre la institución.

- Gestión permanente sobre los llamados "ítems gestionables" del Subtítulo 21, es decir, horas extraordinarias, viático, reemplazos cortos, en el sentido de optimizar el uso de estos recursos, sin descuidar la función asistencial. 
Tabla 2. Indicadores de Gestión Financiera

\begin{tabular}{|c|c|c|c|c|c|c|c|}
\hline & Indicador & 2006 & 2007 & 2008 & 2009 & 2010 & Promedio \\
\hline 8 & $\begin{array}{l}\text { Ingresos de } \\
\text { operación } \\
\text { devengados }\end{array}$ & $\begin{array}{c}32098 \\
170\end{array}$ & $\begin{array}{c}36431 \\
528\end{array}$ & $\begin{array}{c}41862 \\
591\end{array}$ & $\begin{array}{c}53500 \\
060\end{array}$ & $\begin{array}{c}59404 \\
507\end{array}$ & $\begin{array}{c}44659 \\
371\end{array}$ \\
\hline 9 & $\begin{array}{l}\text { Ingresos propios } \\
\text { sobre ingresos } \\
\text { devengados (\%) }\end{array}$ & 0,1 & 0,1 & 0,1 & 0,0 & 0,0 & 0,1 \\
\hline 10 & $\begin{array}{l}\text { Ejecución del } \\
\text { subtítulo } 21(\%)\end{array}$ & 100,0 & 100,1 & 100,0 & 110 & 106,1 & 103,2 \\
\hline 11 & $\begin{array}{l}\text { Deuda sobre } \\
\text { subtítulo } 22(\%)\end{array}$ & 2,9 & 0,0 & 10,5 & 14,2 & 22,7 & 10,1 \\
\hline 12 & $\begin{array}{l}\text { Ejecución del } \\
\text { subtítulo } 22 \text { (\%) }\end{array}$ & 94,0 & 90,5 & 100,0 & 107,4 & 111,7 & 100,7 \\
\hline 13 & $\begin{array}{l}\text { Ejecución del } \\
\text { subtítulo } 21 \text { más } \\
\text { ejecución del } \\
\text { subtítulo } 22\end{array}$ & $\begin{array}{c}29677 \\
374\end{array}$ & $\begin{array}{c}35466 \\
173\end{array}$ & $\begin{array}{c}42188 \\
326\end{array}$ & $\begin{array}{c}51529 \\
841\end{array}$ & $\begin{array}{c}56829 \\
274\end{array}$ & $\begin{array}{c}43138 \\
198\end{array}$ \\
\hline 14 & $\begin{array}{l}\text { Rendimiento } \\
\text { del gasto } \\
\text { operacional (\%) }\end{array}$ & 67,0 & 60,3 & 55,3 & 50,5 & 42,9 & 55,2 \\
\hline 15 & $\begin{array}{l}\text { Diferencia } \\
\text { ingresos y gastos } \\
\text { devengados de } \\
\text { operación }\end{array}$ & $\begin{array}{c}2420 \\
796\end{array}$ & 965355 & -325735 & $\begin{array}{c}1970 \\
219\end{array}$ & $\begin{array}{c}2575 \\
233\end{array}$ & 1521174 \\
\hline
\end{tabular}

Tabla 3. Indicadores de Gestión de Recursos Humanos

\begin{tabular}{llcccccc}
\hline & \multicolumn{1}{c}{ Indicadores } & 2006 & 2007 & 2008 & 2009 & 2010 & Promedio \\
\hline 16 & $\begin{array}{l}\text { Rendimiento del Subtítulo 21 } \\
\text { (Personas) }\end{array}$ & 7864 & 8508 & 8317 & 9059 & 8432 & 8436 \\
17 & $\begin{array}{l}\text { Rendimiento del Subtítulo 21 } \\
\text { (Hrs. Semanales) }\end{array}$ & 190,3 & 203,2 & 197,0 & 215,0 & 198,4 & 200,8 \\
18 & $\begin{array}{l}\text { Número de días de licencia } \\
\text { sobre dotación efectiva }\end{array}$ & 18,5 & 21,5 & 19,7 & 20,0 & 20,5 & 20,0 \\
\hline
\end{tabular}

- Gestiones para un adecuado desarrollo del ambiente laboral lo que incide sobre la productividad (subdepartamento de desarrollo de las personas).

- Trabajo de grupos y equipos con ayuda de especialistas para superar el stress agudo y el postraumático, post terremoto.

\section{DISCUSIÓN}

Según los lineamientos de la Reforma del Sistema de Salud en Chile, la provisión de prestaciones de salud a la población, se organiza en Servicios de Salud, que basan su accionar en el funcionamiento y articulación de sus establecimiento de la red, para lograr la continuidad de la atención, buscando impacto sanitario y satisfacción usuaria, resguardando la equidad, el acceso, la oportunidad y la protección financiera. 
En este marco se ha consolidando la instalación de la reforma, donde los Servicios de Salud Pública han tenido diferentes niveles de avance, evidenciando logros parciales y totales, a través de indicadores de gestión en salud. Estos indicadores han reportado resultados que han motivado la organización de comisiones y reuniones entre los directivos de los establecimientos y el servicio de salud, lo que permitió fortalecer sus competencias directivas (14) y direccionar las estrategias (15) ha implementar en la red asistencial en beneficio de la población.

En resumen, este artículo permitió evidenciar los indicadores de gestión en salud relevantes, pertenecientes al área asistencial, financiero y de recurso humano, al igual que permite compartir esta experiencia exitosa con la finalidad de fortalecer el papel de la salud pública como herramienta para mejorar la calidad de vida de la población y gestionar la calidad de atención de la red (16). En principio el presente estudio ha permitido en una primera etapa, visualizar las estrategias implementadas por el Servicio de Salud Ñuble derivadas del impacto de los indicadores de gestión en salud durante el periodo 2006 al 2010, confirmando la hipótesis bajo estudio

Agradecimientos: A la Universidad del Bío-Bío y a la Dirección del Servicio de Salud Ñuble.

Conflicto de intereses: Ninguno

\section{REFERENCIAS}

1. Subramanian V, Delgado I, Due L, Karachi I, Vega J. Inequidad de ingreso y autopercepción de salud: un análisis desde la perspectiva contextual de las comunas chilenas. Revista Médica Chile. 2003; 131(3): 321-330.

2. Ministerio de Salud de Chile [Internet]. Balance de Gestión Integral. Servicios de Salud. 2001. Disponible en www.minsal.cl. Consultado abril 2011.

3. Fondo Nacional de Salud [Internet]. Balance presupuestario devengado Servicios de Salud en miles de pesos. 2001. Disponible en: www.fonasa.cl. Consultado enero 2011.

4. Fondo Nacional de Salud [Internet]. Brechas de Equidad y Eficiencia. 2001. Disponible en: www.fonasa.cl. Consultado julio 2011.

5. Fondo Nacional de Salud [Internet]. Facturación Total de los Servicios de Salud. 2001. Disponible en: www.fonasa.cl. Consultado abril 2011.

6. Servicio de Salud Ñuble [Internet]. Rediseño de la Red Asistencial de Ñuble. 2010. Disponible en www.serviciodesaludnuble.cl. Consultado julio 2011.

7. Fondo Nacional de Salud [Internet]. Estimación de Población Beneficiaria Fonasa y proyección censal INE según comunas, servicios de salud y regiones del país. 2001. Disponible en: www.fonasa.cl. Consultado agosto 2011. 
8. Fondo Nacional de Salud [Internet]. Indicadores de Gestión y asignación de Recursos desde Ministerio de Salud. 2001. Disponible en: www.fonasa.cl. Consultado septiembre 2011.

9. Fuentes R, Verdessi D [Internet]. La Reforma de la Salud en Chile, Caminos y Propuestas. Documento de Estudio. 1996. Disponible en www.minsal.cl. Consultado octubre 2011.

10. Salinas H, Erazo M, Reyes A. Indicadores de Gestión de Servicios de Salud Públicos y Asignación de Recursos desde el Ministerio de Salud de Chile. Revista Médica Chile. 2004; 132(12): 1532-1542.

11. Kaplan R, Norton D. El Cuadro de Mando Integral, Balanced Scorecard. Barcelona: Gestión 2000; 2004.

12. Ministerio de Salud [Internet]. Balance de Gestión Integral. Servicios de Salud. 2001. Disponible en www.minsal.cl. Consultado junio 2011.

13. Sánchez H, Baeza C. Readecuación del Sistema de Salud Chileno. Corporación de Promoción Universitaria. Santiago: CPU; 1991.

14. Huerta $P$, Leyton $C$, Saldías $H$. Análisis de las competencias directivas de una red de salud pública. Revista de Salud Pública (Bogotá). 2009; 11(6): 979-987.

15. Báscolo E. Gobernanza de las organizaciones de salud basados en atención primaria de salud. Revista de Salud Pública (Bogotá). 2010; 12sup (1): 8-27.

16. Molero T, Panunzio A, Cruz S, Núñez M, Zambrano M, Parra I, Sánchez J. Gestión de la calidad de atención en laboratorios clínicos de hospitales públicos en Maracaibo, Venezuela. Revista de Salud Pública (Bogotá). 2010; 12(4): 658-668. 